\title{
Direct Correlation of Transmission Electron Microscopy and Optical Microscopy for Study of Fluorescent Nanodiamonds
}

Haotian Wen ${ }^{1}$, Christian Dwyer ${ }^{2}$ and Shery Chang ${ }^{3}$

${ }^{1}$ UNSW, United States, ${ }^{2}$ Electron Imaging and Spectroscopy Tools, United States, ${ }^{3}$ Electron Microscope Unit, UNSW, Sydney, New South Wales, Australia

Fluorescent nanodiamonds (FNDs) are diamond nanoparticles containing light-emitting color centers. Due to their great prospects for applications in biomedicine, diagnosis and sensing, the optical properties of FND have been extensively studied. However, a major challenge is that FND suffers from low uniformity in its optical properties. For example, it has been reported that the quantum efficiency of FND particles of nominally the same size can vary by 10-90\% [1]. The underlying reasons are complex, but are ultimately attributable to the nanoparticles' atomic-scale structure, including particle shape, size, strain and surface atomic structure and chemistry [2]. In order to realize FND in the above mentioned applications, it is necessary to study the relationship between structure and optical properties (1) at the individual particle level, and (2) for statistically meaningful numbers of particles.

Here, our approach is to achieve precise correlation of optical and structural measurements of lightemitting nanoparticles, which requires linking separate measurements taken at length scales which differ by nearly 3 orders of magnitude. To supplement the lack of spatial resolution of optical measurements, electron microscopy, especially high-resolution TEM, is an ideal solution. We note that the existing correlated light and electron microscopy (CLEM) technique [3] has achieved correlated measurements at an approximately sub- $\mu \mathrm{m}$ scale that is better suited for biological cells and structures $\square \square$

Here, by contrast, we demonstrate the direct correlation of confocal photoluminescence (PL) microscopy with TEM imaging of $\sim 100 \mathrm{~nm}$ FND particles, over a large area of $100 \mu \mathrm{m}$ by $100 \mu \mathrm{m}$, containing approximately 12,000 particles. Our analysis of this exceptionally large number of particles allows us to achieve statistical measurements of high accuracy, namely $<3 \%$, which is to be compared to conventional manual analysis of typically $<50$ particles giving only $\sim 15 \%$ accuracy.

The biggest challenge in correlating PL and TEM to study individual nanoparticles is to achieve (unbiased) accuracy in overlaying the two "maps" from these techniques, which involve (1) very different fields of view, and (2) distortions arising from the imperfect instrumentation. Here we compare two different approaches for overcoming this challenge. One relies on fiducial markers provided by the TEM grid, while the other, more sophisticated, method employs the concept of the mutual information between the PL and TEM maps.

FND particles were synthesized by high pressure and high temperature (HPHT) process, followed by 3 $\mathrm{MeV} \mathrm{H}+$ ion beam irradiation, annealing, and acid wash to create nitrogen-vacancy centers (Figure 1). Automated acquisition of TEM images was carried out on an aberration-corrected TEM (Titan, FEI Co.) operated at $80 \mathrm{kV}$, using SerialEM software. A MATLAB program was developed to "stich" the series of TEM images into a montage map covering the whole 100

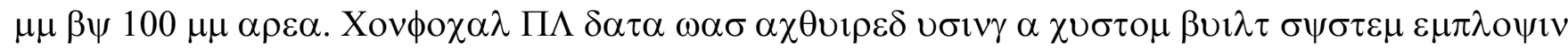

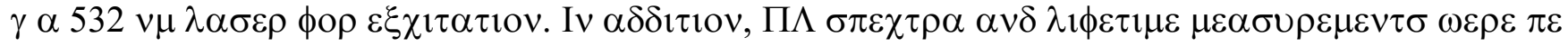

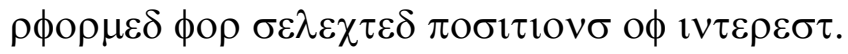


By accurately matching the fluorescence intensities in the PL image with the FNDs nanoparticles in the TEM map, as shown in Fig. 2, we demonstrate that the majority ( $>90 \%$ ) of the nanoparticles or clusters in the studied sample exhibit photoluminescence. Moreover, we find that there is a correlation between the shape of an FND particle and its PL intensity, which is a hitherto unreported effect and contrasts many speculative suggestions in the established literature.
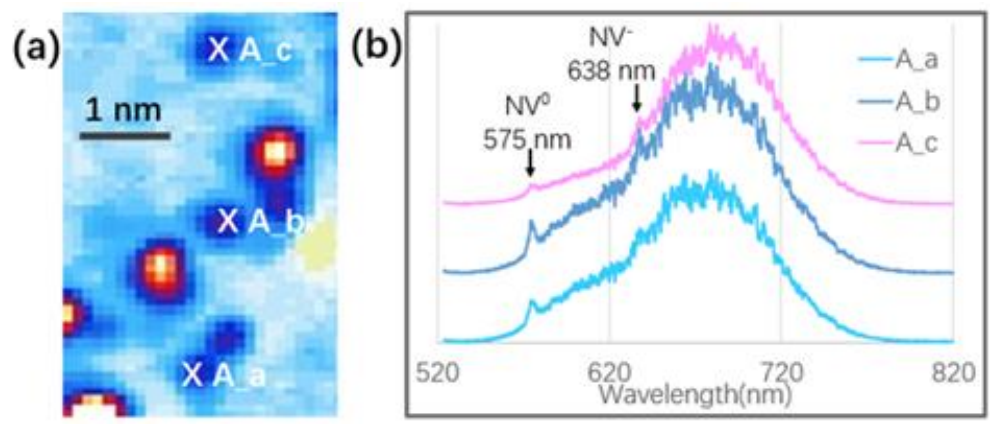

Figure 1. Multi-position PL spectra for the FNDs nanoparticles in the imaging area. (a) The positions (X marks) of where the PL spectra are taken, (b) the corresponding PL spectra, showing the identical ZPL peaks for $\mathrm{N}-\mathrm{V} 0$ and $\mathrm{N}-\mathrm{V}$ - color centers.

(a)

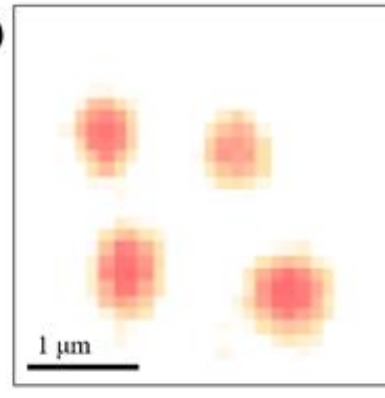

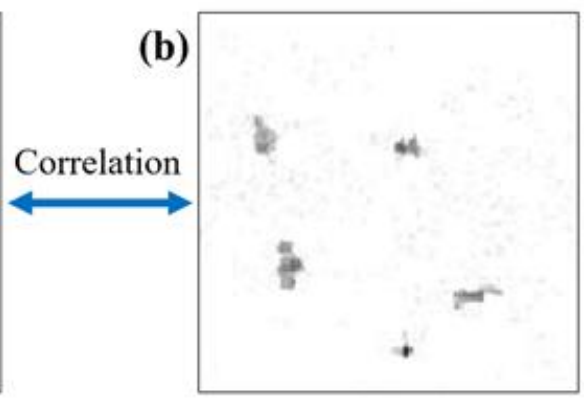

Figure 2. An example (small subregion) correlated PL and TEM data. (a) PL image where orange represents the intensity and spatial extent of the local fluorescent signal. (b) TEM montage. Comparison shows that all particles exhibit fluorescence except for the particle with dark contrast at bottom.

References

[1] A. Mohtashami et al, New Journal of Physics (2013) 15, p. $043017-043039$

[2] J Storteboom et al, Optics Express (2015) 23, 9, pp.11327-11333

[3] P Boer et al, Nature Methods (2015) 12, p. 503-513 